\title{
A new seismic analysis of Alpha Centauri
}

\author{
A. Thoul ${ }^{\star}$, R. Scuflaire, A. Noels, B. Vatovez, M. Briquet, M.-A. Dupret, and J. Montalban \\ Institute of Astrophysics and Geophysics, University of Liège, 17 allée du 6-Août, Sart Tilman (Bât B5c), \\ 4000 Liège 1 , Belgium
}

Received 1 July 2002 / Accepted 10 February 2003

\begin{abstract}
Models of $\alpha$ Cen A \& B have been computed using the masses determined by Pourbaix et al. (2002) and the data derived from the spectroscopic analysis of Neuforge \& Magain (1997). The seismological data obtained by Bouchy \& Carrier $(2001,2002)$ do help improve our knowledge of the evolutionary status of the system. All the constraints are satisfied with a model which gives an age of about 6 Gyr for the binary.
\end{abstract}

Key words. stars: binaries: visual - stars: individual: $\alpha$ Cen - stars: oscillations - stars: evolution

\section{Introduction}

The binary system Alpha Centauri offers a unique opportunity to test our knowledge of stellar physics in solar-type stars other than the Sun. On the one hand, its proximity allows very good determinations not only of its parallax but also of its orbital parameters so that the masses and the luminosities of both components can be determined. On the other hand, effective temperatures and chemical compositions can be obtained through spectroscopic analyses.

A number of theoretical calibrations of this system have already been carried out. The reader will find references to earlier works in the publications of Guenther \& Demarque (2000) and Morel et al. (2000). Very recently, a new and very precise analysis by Pourbaix et al. (2002) has led to new and strong constraints on the masses. Alpha Cen A thus becomes an excellent candidate for asteroseismological studies since solarlike $p$-mode oscillations have recently been discovered from ground-based analysis (Bouchy \& Carrier 2001; Carrier et al. 2002; Bouchy \& Carrier 2002).

In a very recent calibration and seismic analysis, Thévenin et al. (2002) have found that the asteroseismological constraints, namely the large and small frequency spacings, required a slight decrease in the mass assigned by Pourbaix et al. (2002) to $\alpha$ Cen A. Their model reproduces the large and small frequency spacings although the fit in the frequencies themselves is not achieved.

Using an entirely new evolution code, which does not yet include diffusion of chemical elements, we find models for the $\alpha$ Cen binary system which agree not only with all the non-asteroseismic constraints, including the masses and the

Send offprint requests to: A. Thoul, e-mail: thoul@astro.ulg.ac.be

^ Chercheur Qualifié au Fonds National de la Recherche Scientifique, Belgium. requirement of the same age for both components, but also with the asteroseismic constraints.

\section{Observational constraints}

Using data from different sources, Pourbaix et al. (2002) have recently improved the precision of the orbital parameters of $\alpha$ Cen. They have adopted the parallax derived by Söderhjelm (1999), $\varpi=747.1 \pm 1.2 \mathrm{mas}$, and give for the masses $M_{A} / M_{\odot}=1.105 \pm 0.0070$ and $M_{B} / M_{\odot}=0.934 \pm$ 0.0061 .

From their spectroscopic analysis of the $\alpha$ Centauri system, Neuforge \& Magain (1997) have determined the effective temperature, the gravity and the metallicity of both components. The effective temperatures are given by $T_{\text {eff, } A}=5830 \pm 30 \mathrm{~K}$ and $T_{\text {eff }, B}=5255 \pm 50 \mathrm{~K}$. The gravities are given by $\log g_{A}=$ $4.34 \pm 0.05$ and $\log g_{B}=4.51 \pm 0.08$. Since the luminosity can be expressed in terms of the effective temperature and of the gravity through the relation

$\log L / L_{\odot}=\log M / M_{\odot}+4 \log T_{\text {eff }} / T_{\text {eff } \odot}-\log g / g_{\odot}$,

the error box in the $\left(\log T_{\text {eff }}, \log g\right)$ plane appears as a parallelogram in the HR diagram.

For the metallicity, they obtained $[\mathrm{Fe} / \mathrm{H}]_{A}=0.25 \pm 0.02$ and $[\mathrm{Fe} / \mathrm{H}]_{B}=0.24 \pm 0.03$. To deduce $Z / X$, we assume that this ratio is proportional to the abundance ratio $\mathrm{Fe} / \mathrm{H}$ and we adopt the solar value $(Z / X)_{\odot}=0.023$ with an uncertainty of $10 \%$, given by Grevesse \& Sauval (1998). We then have the same value for both components of $\alpha$ Cen: $Z / X=0.040 \pm 0.005$. However, Grevesse \& Sauval (2002) now favour a lower value $(Z / X)_{\odot}=0.0209$ with the same uncertainty. With this solar value, we would have $Z / X=0.037 \pm 0.004$. It seems safe to say that $Z / X$ is between 0.033 and 0.045 .

The above non-asteroseismic constraints are summarized in Table 1. 
Table 1. Non-asteroseismic constraints.

\begin{tabular}{lcc}
\hline \hline & $A$ & $B$ \\
\hline$M / M_{\odot}$ & $1.105 \pm 0.0070$ & $0.934 \pm 0.0061$ \\
$\log T_{\text {eff }}$ & $3.7657 \pm 0.0022$ & $3.7206 \pm 0.0041$ \\
$\log g$ & $4.34 \pm 0.05$ & $4.51 \pm 0.08$ \\
$Z / X$ & \multicolumn{2}{c}{$0.039 \pm 0.006$} \\
\hline
\end{tabular}

\section{The models}

A number of evolutionary sequences have been computed from the main sequence with CLÉS (Code Liégeois d'Évolution Stellaire). We use the CEFF equation of state (Christensen-Dalsgaard \& Däppen 1992). The opacities are those of the Lawrence Livermore National Laboratory (Iglesias \& Rogers 1996) completed with the Alexander \& Ferguson (1994) opacities at low temperature, both tables being smoothly joined in the temperature range defined by $3.95<\log T<4.10$. The nuclear reaction rates are from Caughlan \& Fowler (1988) and the screening factor comes from Salpeter (1954). The boundary conditions at the photosphere are deduced from Kurucz (1998) models. We use the mixing length theory of convection. The convection parameter $\alpha=\ell / H_{P}$ is left free and is used to force the evolutionary track through the error box around the current position of the star in the HR diagram. The suitable value of $\alpha$ depends on the initial chemical composition. It turns out that, for a given composition, we can always use the same value of $\alpha$ for both components, so that the equality $\alpha_{A}=\alpha_{B}$ comes out naturally and is not to be considered as a supplementary constraint. The code does not include diffusion.

We have computed a number of models for both components, with different initial chemical compositions, defined by $(X, Z)$, and convection parameter $\alpha$. We require that both components of the binary system reach their respective positions in the HR-diagram at the same age. This requirement of simultaneity determines a line in the $(X, Z)$-diagram to the right of which the $\mathrm{B}$ component reaches its observed position in the HR-diagram after the A component has already left its own observed position (see Fig. 1). In addition, the chemical evolution of the Galaxy suggests a correlation between $X$ and $Z$. The relation $X=0.76-3 Z$ which has been used by Pols et al. (1998) is also shown in Fig. 1. Although such a relation may have a statistical significance, it may be hazardous to strictly adhere to it in a particular case. These constraints, together with the constraint on the $Z / X$ value, delimit a permitted area in the $(X, Z)$ diagram.

\section{Asteroseismology of $\alpha$-Centauri}

Bouchy \& Carrier (2001), Carrier et al. (2002), and Bouchy \& Carrier (2002) have recently observed the oscillation spectrum of $\alpha$ Cen A with the CORALIE spectrograph. In the first paper, they observed the star during 5 nights, and were able to identify some $l=0$ and $l=1$ modes, but the resolution was too low to identify separately the $l=0$ and the $l=2$ modes. In their next run of observations, they observed the star during 11 nights,

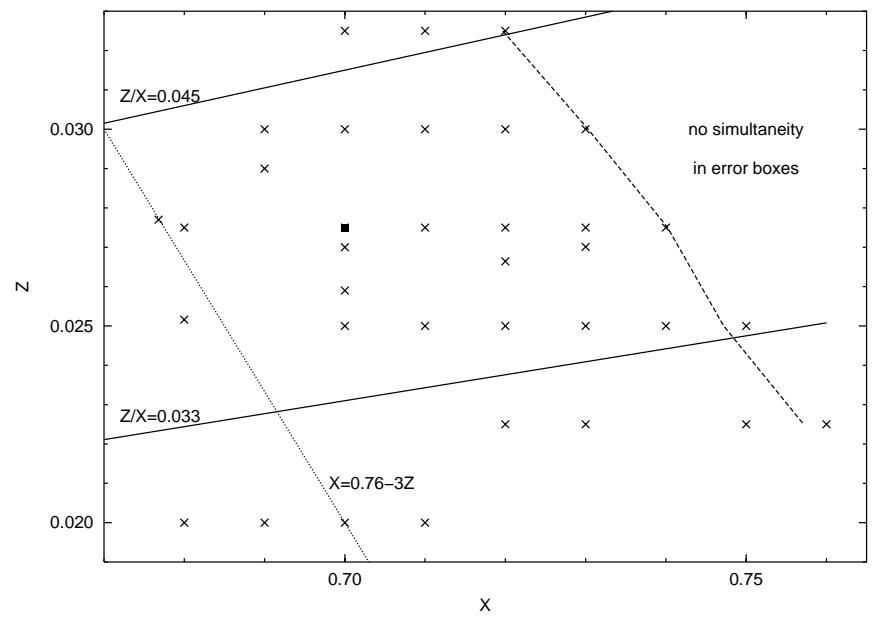

Fig. 1. Constraints on the initial chemical composition coming from the spectroscopy (solid line) and from the simultaneity condition (dashed line). The dotted line represents the chemical evolution of the Galaxy. The crosses indicate the different compositions for which evolution sequences have been computed. The black square represents the model discussed in detail in this paper.

and were able to identify many $l=0, l=1$ and $l=2$ modes. They could not resolve the $l=3$ modes. Their latest results are summarized in Table 2. They obtain an uncertainty of $0.46 \mu \mathrm{Hz}$ on these frequencies, but add that an error of $\pm 1.3 \mu \mathrm{Hz}$ could have been introduced for some of the frequencies due to aliases. For this reason, we will adopt this last value for the observational error bars.

We have calculated the oscillation frequencies of $\alpha$ Cen $\mathrm{A}$ using a standard adiabatic code (Boury et al. 1975), for a grid of models where the mass $M_{A}$ is fixed at the value determined by Pourbaix et al. (2002), i.e., $M_{A}=1.105 M_{\odot}$. The only free parameters are $\alpha, X$, and $Z$, and the last two can only vary within the permitted area illustrated in Fig. 1. The models we have explored are shown as crosses in Fig. 1. The models which fall outside of the permitted area were explored for reason of completeness only.

We calculate the modes $l=0, l=1$ and $l=2$ for $n=15$ to 25 . We then calculate the large frequency spacings $\Delta v_{l, n}=$ $v_{l, n}-v_{l, n-1}$ and the small frequency spacings $\delta v_{0, n}=v_{l, n}-$ $v_{l+2, n-1}$, and their averages over $n=15$ to 25 .

\section{Discussion and conclusions}

We will discuss the results corresponding to one of our best solutions (see Table 3), shown as a black square in Fig. 1. Let us notice that for the same physics, a solar calibration gives $\alpha=1.77$. The trajectories in the HR diagram for the two components of the system are shown in Fig. 2. The cross on the A component track in the HR-diagram shows the model retained for our discussion. We list the frequencies we have obtained for this particular model in Table 2. Finally, we show in Figs. 3 and 4 the results for the large and the small frequency spacings. The age of this particular model is $t=6.4 \mathrm{Gyr}$. The position of the B component in the HR diagram at the same age is also marked with a cross in Fig. 2. 
Table 2. Mode frequencies (in $\mu \mathrm{Hz}$ ) of $\alpha$ Cen A.

\begin{tabular}{ccccccc}
\hline \hline \multicolumn{7}{c}{ Observations $^{a}$} \\
& $l=0$ & $l=1$ & $l=2$ & $l=0$ & $l=1$ & $l=2$ \\
\hline$n=15$ & & & 1833.1 & 1730.5 & 1778.5 & 1828.0 \\
$n=16$ & 1841.3 & 1887.4 & 1934.9 & 1834.9 & 1882.8 & 1932.6 \\
$n=17$ & & 1991.7 & 2041.5 & 1939.3 & 1987.7 & 2038.1 \\
$n=18$ & & 2095.6 & 2146.0 & 2044.4 & 2093.7 & 2144.7 \\
$n=19$ & 2152.9 & 2202.8 & 2251.4 & 2150.8 & 2200.4 & 2251.7 \\
$n=20$ & 2258.4 & 2309.1 & 2358.4 & 2257.5 & 2307.5 & 2358.7 \\
$n=21$ & 2364.2 & 2414.3 & 2464.1 & 2364.2 & 2414.4 & 2465.9 \\
$n=22$ & 2470.0 & 2519.3 & 2568.5 & 2471.0 & 2521.5 & 2573.1 \\
$n=23$ & 2573.1 & 2625.6 & & 2578.0 & 2629.0 & 2681.0 \\
$n=24$ & 2679.8 & 2733.2 & 2782.9 & 2685.5 & 2736.9 & 2789.2 \\
$n=25$ & 2786.2 & 2837.6 & 2887.7 & 2793.4 & 2845.2 & 2897.6 \\
\hline
\end{tabular}

${ }^{a}$ Bouchy \& Carrier (2002).

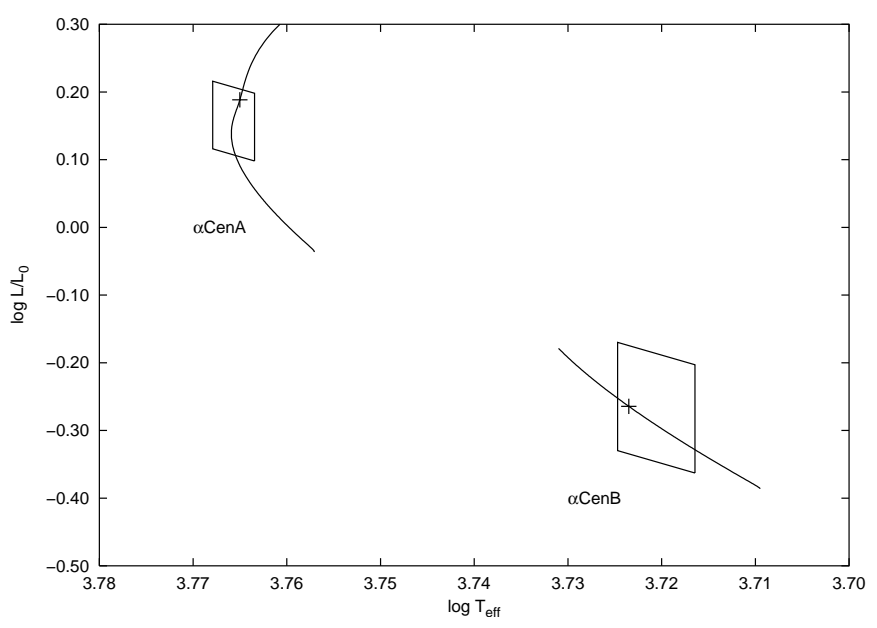

Fig. 2. Evolutionary tracks in the HR diagram for the sequences obtained with $X=0.70, Z=0.0275$, and $\alpha=1.8$. The $1 \sigma$ error boxes on $\log L / L_{\odot}$ and $\log T_{\text {eff }}$ for both components appear as parallelograms. The crosses denote the model retained for our discussion.

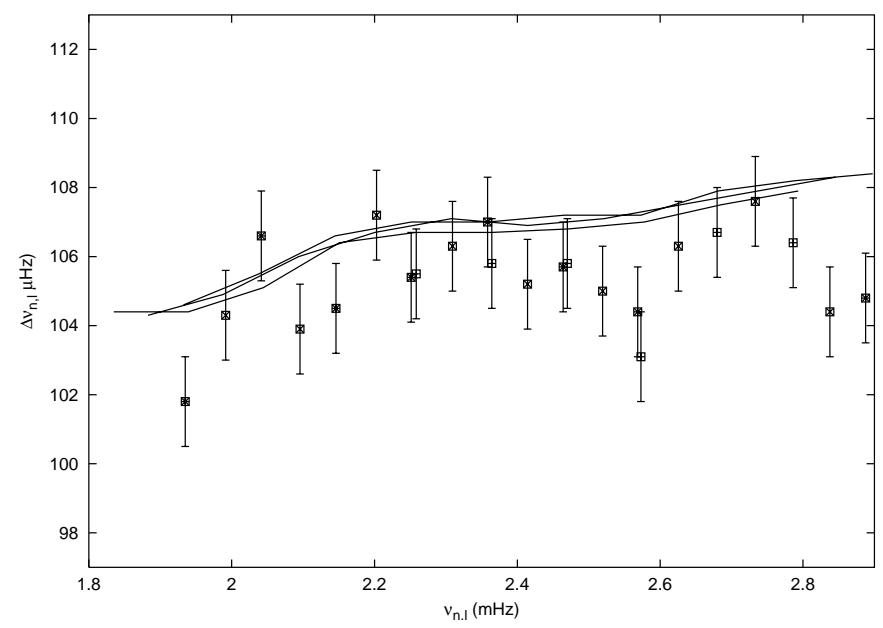

Fig. 3. Large frequency spacing as a function of the frequency. The symbols indicate the values obtained by Bouchy \& Carrier (2002), with error bars of \pm 1 . $\mu \mathrm{Hz}$. The three lines correspond to the model shown as a cross in Fig. 2, for $l=0, l=1$ and $l=2$.
This model satisfies all the non-asteroseismic constraints listed in Table 1. Moreover, the two components of the system have the same age. As to the asteroseismic constraints, the average of the large spacings is slightly larger than the one obtained from the observations, but those large spacings remain within the error bars, as shown in Fig. 3. The values for the small spacings fall within the error bars as well, as shown in Fig. 4. Finally, we note that this solution was found for a value of the mixing-length parameter $\alpha$ very close to the solar value.

The value of the average small spacing $\left\langle\delta v_{0}\right\rangle$ is sensitive to the structure of the core of the star, and therefore to its chemical composition. Let us note that our retained model does not have a convective core, but it nevertheless leads to an excellent agreement for the small frequency spacings. The value of $\left\langle\delta v_{0}\right\rangle$ increases as we lower the value of $X$. But all the models which lie on a curve more or less parallel to the dashed curve in Fig. 1 provide the same value for $\left\langle\delta v_{0}\right\rangle$. We are unable, given the resolution of the observations available, to discriminate between those solutions. To go one step further we would need additional observational data, such as the $l=3$ modes, or even better, the oscillation frequencies of $\alpha$ Cen B.

We have been able with our model to reproduce quite well, and certainly within the observational error bars, both the large and the small frequency spacings observed by Bouchy et al. with the CORALIE spectrograph for $\alpha$ Cen A, while respecting all the non-asteroseismic constraints of the system. We must however be very careful with the interpretation of the results, since we have not included the diffusion of helium and other heavy elements in our stellar evolution code. It is well known that the inclusion of diffusion has greatly improved the agreement between solar models and observations (see, e.g., Bahcall \& Pinsonneault 1995; Morel et al. 1997). However, several papers have examined the influence of diffusion on models of $\alpha$ Cen A (Edmonds et al. 1992; Turcotte $\&$ Christensen-Dalsgaard 1998) and they have shown that diffusion of helium has no detectable effect on the frequencies of the low $l$-degree high-order $p$-modes, and therefore no effect on the large and the small frequency separations, in the frequency range of the spectrum of $\alpha$ Cen $A$. But it has a substantial 
Table 3. Our model.

\begin{tabular}{lcc}
\hline \hline & $A$ & $B$ \\
\hline$M / M_{\odot}$ & 1.105 & 0.934 \\
$L / L_{\odot}$ & 1.54 & 0.544 \\
$T_{\text {eff }}$ & $5821 \mathrm{~K}$ & $5291 \mathrm{~K}$ \\
$X$ & \multicolumn{2}{c}{0.70} \\
$Z$ & \multicolumn{2}{c}{0.0275} \\
$\alpha$ & \multicolumn{2}{c}{1.8} \\
age & \multicolumn{2}{c}{$6.41 \mathrm{Gyr}$} \\
\hline
\end{tabular}



Fig. 4. Small frequency spacing $\delta v_{0}$ as a function of the frequency. The symbols represent the values obtained by Bouchy \& Carrier (2002), with error bars of $\pm 1.3 \mu \mathrm{Hz}$. The line corresponds to the model shown as a cross in Fig. 2.

effect on the age and on the initial composition. According to Edmonds et al. (1992) diffusion lowers the age of the star by about $10 \%$, and it increases the initial helium abundance to $Y=0.30$.

In their very recent paper, Thévenin et al. (2002) propose a model for $\alpha$ Cen A which gives acceptable values for the large and the small frequency spacings, but they had to change the mass $M_{A}$ of the star. In addition, their frequencies differ from the observed frequencies by $\approx 30 \mu \mathrm{Hz}$. It is well-known that the exact values of the frequencies themselves depend on the details of the star's atmosphere, where the pulsation is non-adiabatic. A discrepancy of $30 \mu \mathrm{Hz}$ seems rather large, however, since typical discrepancies for solar $p$-mode frequencies are smaller than $10 \mu \mathrm{Hz}$ (see, e.g., Guenther et al. 1996; Guenther \& Demarque 1997). We show in Fig. 5 the differences between the calculated frequencies and the observed frequencies for our model. We see that these differences display a pattern similar to what is found for the Sun (Guenther et al. 1996; Guenther \& Demarque 1997).

The evolution code used by Thévenin et al. (2002) differs from ours in two ways: they use the Canuto \& Mazitelli (1991, 1992) theory of convection (hereafter CM) below an optical depth $\tau=20$ while we use the basic Böhm-Vitense (1958) mixing-length theory of convection (hereafter MLT), and they include the effects of helium diffusion. Morel et al. (2000) obtain very different ages for the $\alpha$ Cen system when

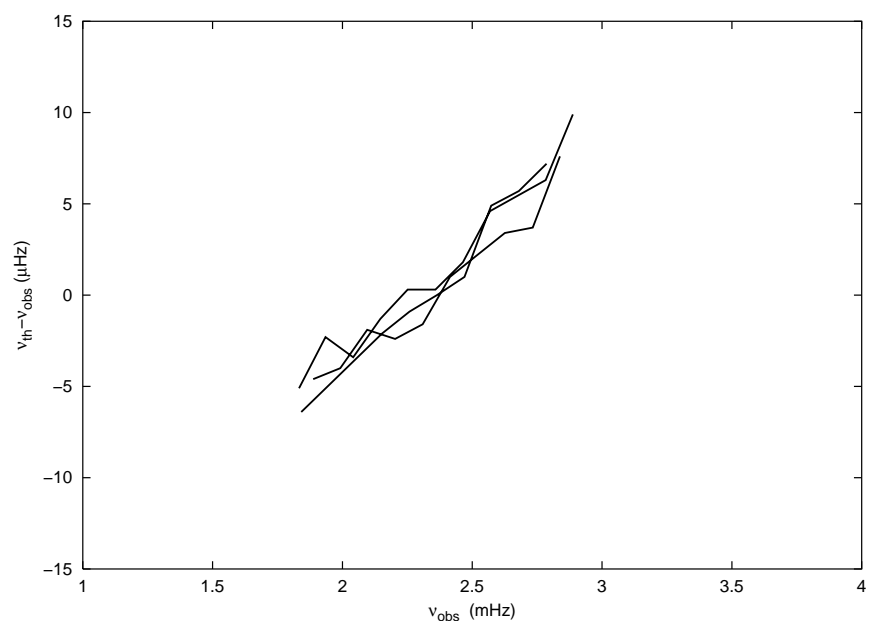

Fig. 5. Differences between the calculated frequencies and the observed frequencies for our model.

using the CM or the MLT theories of convection: $4086 \mathrm{Myr}$ and $2710 \mathrm{Myr}$ respectively. In fact, changing the treatment of the convection, while keeping all other stellar parameters the same, will affect the value of the effective temperature at a given age (or luminosity). Therefore, in order to go through a given position in the HR-diagram, it is necessary to modify, e.g., the helium abundance. Morel et al. (2000) had to use $Y=0.284$ in MLT models, and $Y=0.271$ in CM models. This of course changes considerably the age of the star. On the other hand, diffusion only changes the initial helium abundance by about $0.5 \%$ (Turcotte \& Christensen-Dalsgaard 1998), and the age by 0.4 Gyr (Edmonds et al. 1992). Furthermore, the effects of microscopic diffusion could be even reduced if turbulent mixing is taken into account (Turcotte et al. 1998). In addition, Thévenin et al. (2002) use observations different from the ones we use to calibrate their models (Morel et al. 2000). The effective temperature they use for $\alpha$ Cen A is $40 \mathrm{~K}$ lower than ours. This will also affect the luminosity. Moreover, they use a rather smaller value for the metallicity $[\mathrm{Fe} / \mathrm{H}]=0.20$ instead of our $[\mathrm{Fe} / \mathrm{H}]=0.25$. The discrepancies between the results of Thévenin et al. (2002) and the results we present here could result from those differences. It would of course be very useful to compare the results of different stellar evolution codes in a more consistent and systematic manner.

Acknowledgements. We thank J. Christensen-Dalsgaard who kindly supplied us with the code for the computation of the CEFF equation of state. This work has been supported by the PRODEXESA/Contract\#15448/01/NL/SFe(IC).

\section{References}

Alexander, D. R., \& Ferguson, J. W. 1994, ApJ, 437, 879

Bahcall, J. N., \& Pinsonneault, M. H. 1995, Rev. Mod. Phys., 67, 781

Böhm-Vitense, E. 1958, Z. Astrophys., 54, 114

Bouchy, F., \& Carrier, F. 2001, A\&A, 374, L5

Bouchy, F., \& Carrier, F. 2002, A\&A, 390, 205

Boury, A., Gabriel, M., Noels, A., Scuflaire, R., \& Ledoux, P. 1975, A\&A, 41, 279

Canuto, V. M., \& Mazitelli, I. 1991, ApJ, 370, 295 
Canuto, V. M., \& Mazitelli, I. 1992, ApJ, 389, 729

Carrier, F., Bouchy, F., Meynet, G., et al. 2002, in Radial and Nonradial Pulsations as Probes of Stellar Physics, ed. C. Aerts, T. R. Bedding, \& J. Christensen-Dalsgaard, ASP Conf. Ser., 259, 460

Caughlan, G. R., \& Fowler, W. A. 1988, Atomic data and nuclear data tables, 40, 283

Christensen-Dalsgaard, J., \& Däppen, W. 1992, A\&ARv, 4, 267

Edmonds, P., Cram, L., Demarque, P., Guenther, D. B., \& Pinsonneault, M. H. 1992, ApJ, 394, 313

Grevesse, N, \& Sauval, A. J. 1998, Space Sci. Rev., 85, 161

Grevesse, N., \& Sauval, A. J. 2002, private communication

Guenther, D. B., Kim, Y.-C., \& Demarque, P. 1996, ApJ, 463, 382

Guenther, D. B., \& Demarque, P. 1997, ApJ, 484, 937

Guenther, D. B., \& Demarque, P. 2000, ApJ, 531, 503

Iglesias, C. A., \& Rogers, F. J. 1996, ApJ, 464, 943

Kurucz, R. L. 1998, http: //cfaku5 . harvard.edu/grids.html
Morel, P., Provost, J., \& Berthomieu, G. 1997, A\&A, 327, 349

Morel, P., Provost, J., Lebreton, Y., Thévenin, F., \& Berthomieu, G. 2000, A\&A, 363, 675

Neuforge-Verheecke, C., \& Magain, P. 1997, A\&A, 328, 261

Pols, O. R., Schröder, K.-P., Hurley, J. R., Tout, C. A., \& Eggleton, P. P. 1998, MNRAS, 298, 525

Pourbaix, D., Neuforge, C., \& Noels, A. 1999, A\&A, 344, 172

Pourbaix, D., Nidever, D., McCarthy, C., et al. 2002, A\&A, 386, 280

Salpeter, E. E. 1954, Austral. J. Phys., 7, 373

Söderhjelm, S. 1999, A\&A, 341, 121

Thévenin, F., Provost, J., Morel, P., et al. 2002, A\&A, 392, L9

Turcotte, S., \& Christensen-Dalsgaard, J. 1998, in Structure and Dynamics of the Interior of the Sun and Sun-like Stars, ESA SP-418, ed. S. G. Korzennik, \& A. Wilson (Noordwijk: ESA Publications Division), 407

Turcotte, S., Richer, J., \& Michaud, G. 1998, ApJ, 504, 559 